\title{
Experimental research on hydrodynamic instabilities during condensation of the pro-ecological refrigerant R1234yf in tubular minichannels
}

\author{
Waldemar Kuczyński ${ }^{1, *}$, and Aleksander Denis ${ }^{1}$ \\ ${ }^{1}$ Technical University of Koszalin, Department of Energy, av. Raclawicka 15-17, PL 75-620 Koszalin, Poland
}

\begin{abstract}
The following paper presents the results of preliminary experimental research on the influence of instabilities of a hydrodynamic type on the condensation phase change process in tubular minichannels. The research was focused on a new pro-ecological refrigerant, R1234yf, intended as a substitute for R134a that currently is being phased out. The flow condensation phase change process was investigated for both steady and un-steady conditions in singular tubular minichannels with an internal diameter $d=\{1,44 ; 2,30 ; 3,30\}$ $\mathrm{mm}$. The scope of the analysis of the experimental data covered an estimation of propagation velocities for both pressure and temperature instabilities as well as the shrinkage of the condensation zone. The results were also compared with the previous results obtained for the flow condensation phase change of R134a refrigerant in tubular minichannels with the same internal diameters.
\end{abstract}

\section{Introduction}

The refrigerant is a substance or a blend that circulates within a closed loop. It absorbs heat while evaporating at a low temperature and pressure, and it rejects heat while condensing at a high temperature and pressure. Refrigerants are used in fridges, freezers, air conditioners, heat pumps and Organic Rankine Cycles. Due to an unequivocal impact on the environment, refrigerants are subjected to many international regulations, such as:

- The Montreal Protocol on Substances that Deplete the Ozone Layer (1987),

- The United Nation Framework Convention on Climate Change (1992),

- The Regulation (EU) No 517/2014 of the European Parliament and of the Council of 16 April 2014 on Fluorinated Green-House Gases.

The first agreement introduced the Ozone Depletion Potential (ODP) indicator that illustrates the negative impact of each refrigerant on the ozone layer. As a result of this agreement, all chlorine based refrigerants $(\mathrm{ODP}>0)$ were banned from use. The second agreement introduced the Global Warming Potential (GWP) indicator that illustrates a contribution of each refrigerant to the global warming effect with carbon dioxide as a reference pollutant $\left(\mathrm{GWP}_{\mathrm{CO} 2}=1\right)$. The EU Regulation No $517 / 2014$, however, sets a roadmap for the phase-out of the so-known as fluorinated green-house gases - in particular hydro-fluoro-hydrocarbons (HFC), per-fluorohydrocarbons (PFC), sulphur hexafluoride and other fluorinated refrigerants. It aims at a $66 \%$ reduction in emission of fluorinated green-house gases (base level 2010). One of those green-house gases is the R134a refrigerant that is currently commonly used in vehicle air conditioning systems and heat pumps. According to the roadmap, it has already been banned from use in brand new installations since January the 1st 2017. Currently, the replacement for the R134a refrigerant are R1234yf for vehicle air conditioning systems and R1234ze(E) for heat pumps. The new refrigerants have significantly lower GWP indicators, $\mathrm{GWP}_{\mathrm{R} 1234 \mathrm{yf}}=4, \mathrm{GWP}_{\mathrm{R} 1234 \mathrm{ze}(\mathrm{E})}=7$, they are non-toxic hydro-fluoro-olefins (HFO), but contrary to $\mathrm{R} 134 \mathrm{a}$, they are officially considered as slightly flammable $[1,2,3,4,5]$.

All in all, from the engineering perspective, the main conclusion of those regulations is that nowadays the priorities in the refrigerant slection process look as follow:

- environmental impact,

- operational safety,

- thermodynamic efficiency,

- other technical aspects (like the corresponding type of oil, etc.),

- economics.

The following research was done using minichannels. According to Kandlikar [6], they have an ID (internal diameter) between 0.2 and $3 \mathrm{~mm}$, and according to Mehendale [7] between 1 and $6 \mathrm{~mm}$. Wider channels are known asknown as conventional ones, and smaller are known asknown as meso, micro or nano channels depending on their ID.

The flow through conventional channels is governed by inertia forces. In minichannels, however, the flow is governed by capillary forces like viscosity and surface tension $[8,9]$.

Minichannels allow one to shrink heat exchangers and to reduce the coolant mass in the system (in other

\footnotetext{
Corresponding author: waldemar.kuczynski@tu.koszalin.pl
} 
words, F-gas emission in the case of leakage). Moreover, much higher heat flux density enables a rapid development of high power density electronics. Although it comes at the expense of higher hydraulic losses, only in the case of a high flow rate the pressure penalty exceeds the heat transfer gain [10]. The heat transfer can be further enhanced using the microfins [11], parallel channels configuration [12] or by operating in an unstable two-phase region [13].

Instabilities are inherently connected with phase changes, evaporation and condensation, and the twophase flow [14]. The first discovered instability was the Ledinegg's flow excursion [15] - the pressure drop versus flow rate (or the $\mathrm{N}$-curve) for heated channels may have up to 3 different points of operation. The occurrence of instabilities depends on the flow rate, heat flux and medium conditions at the inlet (like quality, superheating or subcooling) [16] and system characteristics (like the presence of internal compressible volumes) [17]. The instabilities mainly affect flow distribution in parallel channels [18], flow pattern [19] and pressure drop [20].

In conclusion, most researchers focus on flow boiling instabilities due to more severe potential consequences connected with an uncontrolled temperature rise (like burn-out or meltdown). Some researchers focus on flow condensation instabilities, some on the thermalhydraulics of mini and microchannels, but only few have investigated flow condensation instabilities in minichannels [21].

The experimental research on the influence of hydrodynamic instabilities on the flow condensation process in minichannels has already been conducted for those refrigerants that are currently being phased-out [22 - 33]. However, the influence of this type of instabilities on the phase change of new pro-ecological refrigerants is not known. It is well known that during the flow condensation of the currently used refrigerants under hydrodynamic instabilities, the instabilities propagate in a wave form. The two-phase mediums are especially susceptible for this phenomenon.

Hydrodynamic instabilities occurring in two- and multi-phase mediums during flow condensation inside minichannels may have a different origin [8]:

- onset of phase-change,

- density variation,

- flow variation,

- pressure variation,

- temperature variation,

- quality variation,

- shock-wave,

- Kelvin-Helmholtz instability due to the interference of surface waves.

Among different instabilities connected to mass flux oscillations, the acoustic wave and condensation front are of a prime importance. Each one of them has a different characteristics, especially the propagation velocity within the medium. Both of them are considered as dissipative and dispersive. Dissipative means that the process is irreversible (generates entropy), thus it does not self-sustain, but decays in time. Dispersive means that the propagation velocity strongly depends on the frequency of induced instabilities.

The condensation front manifests itself as a displacement of a sudden drop of wall temperature, in other words, a shrinkage of the high efficiency condensation zone.

A typical condenser during a steady state operation is supplied with a superheated gas and discharges a subcooled liquid. Therefore, it can be divided into 3 characteristic zones: cooldown of superheated gas, two-phase condensation and sub-cooling of liquid. The size of the condenser is constant; thus, any transient, hydrodynamic instabilities in particular, affects $\backslash$ the distribution of these zones. As a result the performance of the condenser drops, and in the worst case scenario, the whole system may be damaged.

All the wave phenomena are strongly coupled, in other words, an onset of one of them usually triggers an onset of all of them. For example, a dynamic change of the flow rate triggers variations of density, pressure, temperature and quality. This paper aims to characterize the hydrodynamic instabilities present during condensation of the pro-ecological refrigerant R1234yf, both qualitatively and quantitatively.

\section{Apparatus}

The diagram of the apparatus used for the experimental part of the research is shown in FigureFigure 1. Refrigerant leaves the tank in liquid state at a high pressure and room temperature. Then it decreases pressure thanks to expansion valve and evaporates while absorbing ambient heat in the evaporator. The compressor increases the pressure and temperature of the gaseous refrigerant. While leaving the compressor, the refrigerant's stream splits in two: most of the flow returns to the tank via the condenser, where it turns back into a liquid while rejecting heat, and the remaining part (the exact amount is adjusted using the regulation valve) is directed to the test section. At the entrance, there is a cut-valve that induces the instabilities. Its timings are controlled via a controller.

The test section (Figure 2) is a minichannel armed with type $\mathrm{K}$ thermocouples and pressure sensors that is immersed in water bed. To maintain the constant temperature of the cooling water, a water pre-conditioner is used. A residual gaseous refrigerant is condensed in the after cooler. The liquefied refrigerant flows through a flow-meter and returns to the main stream. Analog voltage-based signals from the thermocouples, the flow meter and the pressure sensors are converted to digital ones using A/D adapters and stored using a PC.

Figure 3 shows a sample of a change of flow in time registered using the apparatus, there is a slight delay between the pressure wave and the flow recovery. Just before the cut-valve re-opening, the minichannel is being re-flooded by liquid and after the re-opening the incoming gas encounters a "cork" of stagnant liquid. Moreover, the maximum flow rate after the cut-valve reopening exceeds the steady state flow rate $(\sim 1.85 \mathrm{~kg} / \mathrm{h})$ multiple times, depending on the frequency. 


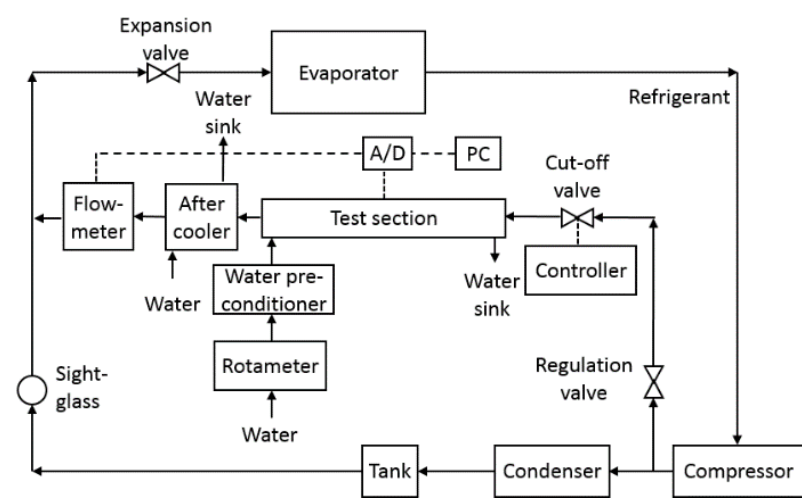

Fig. 1. Diagram of the apparatus.

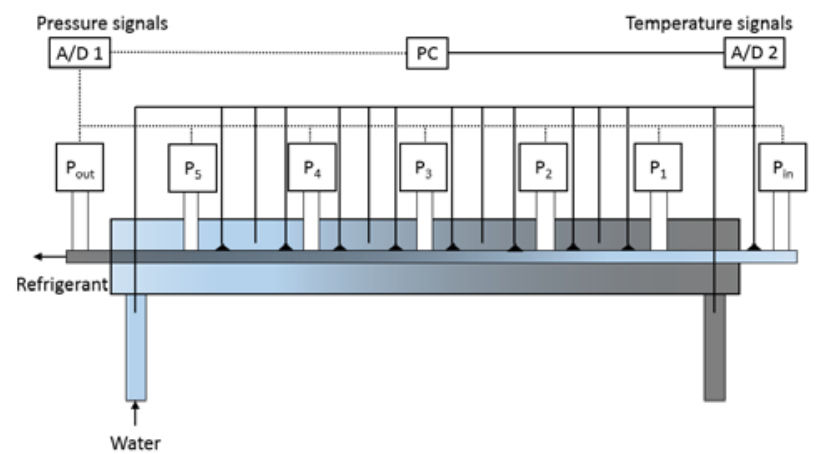

Fig. 2. Diagram of the test section.

Figure 4 represents an evolution of pressure in time registered by multiple sensors installed along the minichannel's length. Right after the cut-valve closure, the pressure suddenly drops, next it recovers to a plateau and after the valve re-opening it increases very rapidly. At first glance, they seem identical, but due to pressure losses the signals are slightly shifted vertically and horizontally due to the pressure wave travelling through the system.

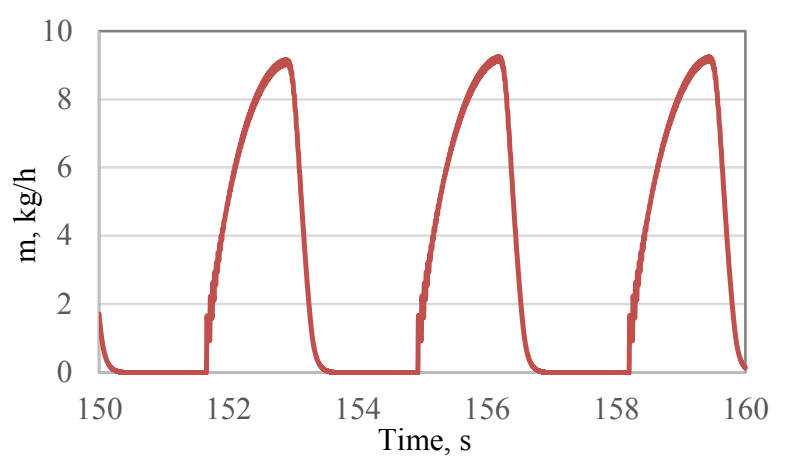

Fig. 3. Sample of flow oscillations.

Figure 5 illustrates temperature oscillations registered by multiple sensors distributed along the channel. The thermal phenomena are much slower than the pressure wave, thus the differences in signals are much easier to observe. Again, the vertical shift is due to the heat transfer between the refrigerant and cooling water, and the horizontal shift is due to the condensation front travelling through the system.

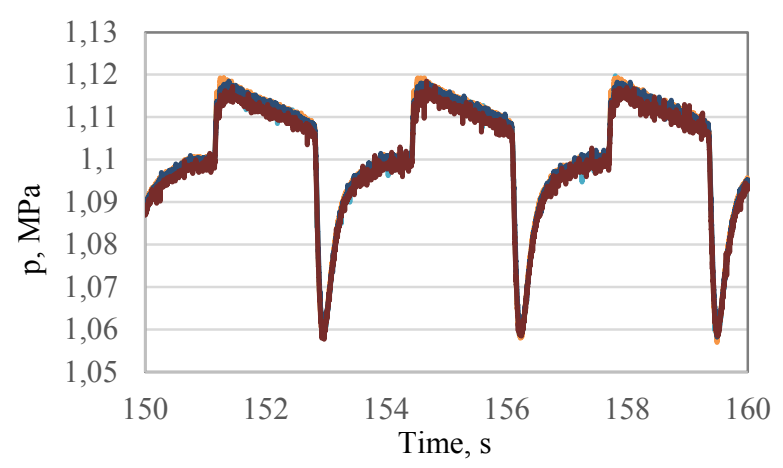

Fig. 4. Sample of pressure oscillations for multiple sensors

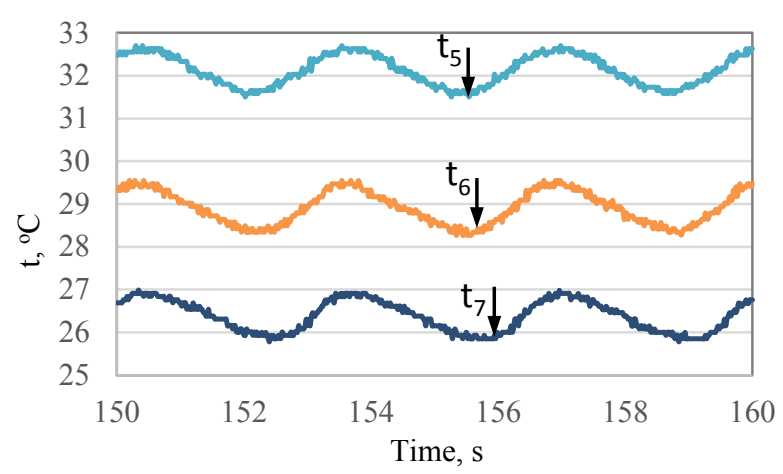

Fig. 5. Sample of temperature oscillations for multiple sensors.

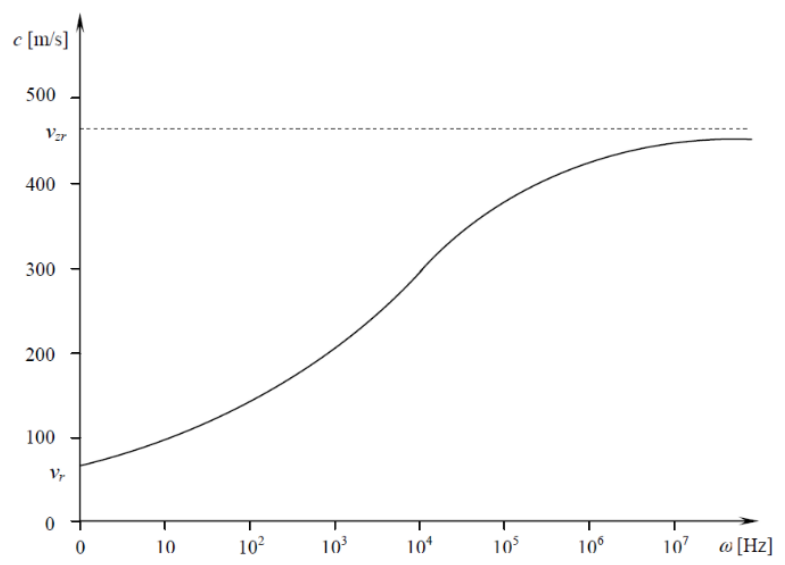

Fig. 6. Pressure wave velocity as a function of frequency for conventional channels [25].

\section{Methodology}

The literature provides a basic theory on the dispersive character of the periodic instabilities, or in other words how the frequency of those disturbances affects the their propagation velocity. The curve (shown in Figure 6) has 2 characteristic asymptotes. The first one is known asknown as an equilibrium velocity:

$$
v_{E}=v(\omega \rightarrow 0)
$$

and it represents a situation when the wave period is longer than the relaxation time (time of re-establishing of the thermodynamic equilibrium) and the pressure wave propagates slower. The second asymptote is known as a frozen velocity: 


$$
v_{F}=v(\omega \rightarrow \infty)
$$

and it represents a situation when the wave period is shorter than the relaxation time and the pressure wave propagates much faster.

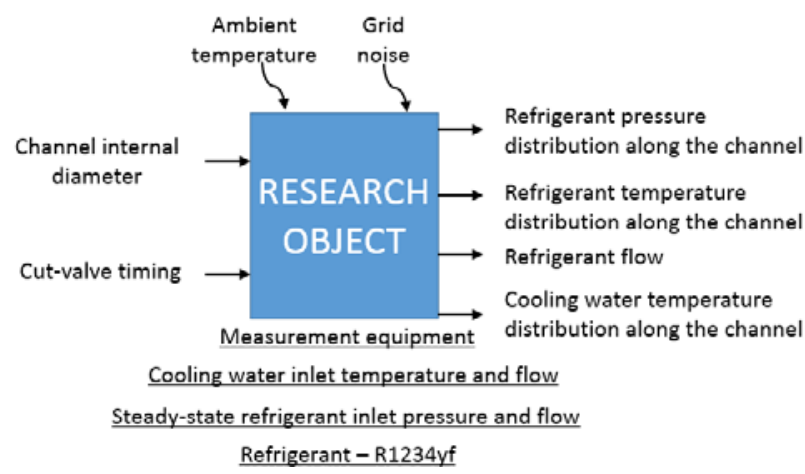

Fig. 7. Research object.

The research object for the experimental part is shown in Figure 7. The internal diameter of the channel and cut-valve timings were the input values. Flow, and pressure and temperature distributions were the output values. The constants of the model were the equipment type, cooling water inlet conditions and steady state refrigerant inlet conditions. Known influences that could not be eliminated were ambient temperature variation during the long lasting experiments and noises coming the complex power supply grid that supplies electricity to the laboratory and noticeably affected the operation of the measurement system. The experimental data was acquired using the DasyLab software.

The methodology of the experimental research part was analogical to the other researchers of this subject, like Teng, Lee, Kuczynski or Bohdal. Signals from multiple sensors (examples shown in Figures 8 and 9 for pressure and temperature, respectively) were compared in order to find a time shift between their responses to the cut-valve operation.

For each frequency, multiple time shifts were averaged in order to minimize the uncertainty of the average value. The distance between sensors was well known, and the final results were obtained using this simple formula - distance over time shift:

$$
\bar{v}=\Delta l / \Delta \bar{\tau}
$$

where:

$\Delta \tau$ - time shift,

$\Delta \mathrm{l}-$ distance between sensors.

An estimation of time shifts (Figures 8 and 9) "by hand" could be arbitrary. Therefore, to eliminate the human factor, a curve fitting procedure was employed. For pressure signals the 4 parameter sigmoid was selected as a reference curve:

$$
y(\tau)=\frac{a}{1+\exp \left(-k *\left(\tau-\tau_{0}\right)\right)}+c
$$

where:

a-gain,

$\mathrm{k}$ - shape parameter,

$\tau_{0}-$ inflection point, $\mathrm{c}$ - initial value.

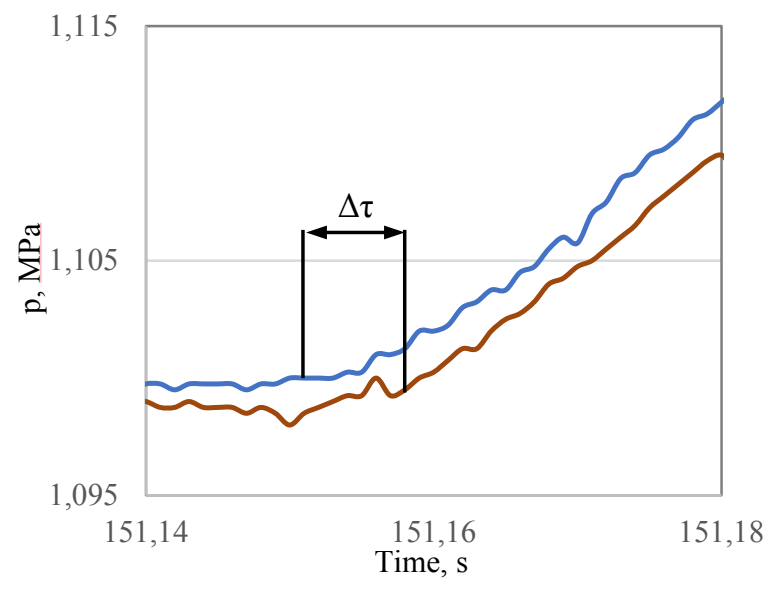

Fig. 8. Time shift between pressure sensors.

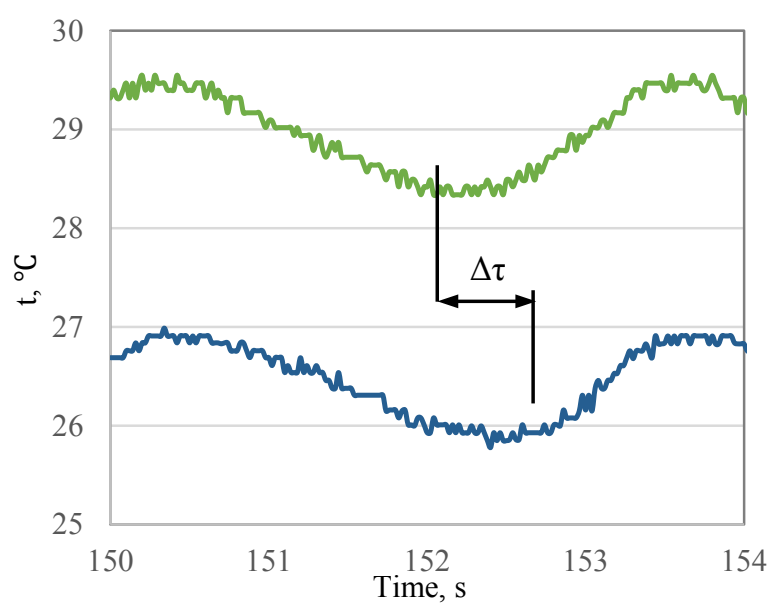

Fig. 9. Time shift between temperature sensors.

For temperature signals, however, the resized and shifted sinusoid was selected as a reference curve:

$$
y(\tau)=a * \sin \left(\tau+\tau_{0}\right)+c
$$

where:

a - resize parameter,

$\tau_{0}$ - phase,

$\mathrm{c}-$ average value.

The curve fitting procedure was done using a combination of Python's libraries such as numpy, pandas, pylab and scipy.

\section{Results}

The scope of the preliminary research covered:

- One refrigerant - R1234yf,

- Three internal diameters $\mathrm{ID}=\{3.3 ; 2.3 ; 1.44 \mathrm{~mm}\}$,

- Single channel configuration,

- Refrigerant mass flux $\mathrm{G}=60 \div 316 \mathrm{~kg} /\left(\mathrm{m}^{2} * \mathrm{~s}\right)$,

- Refrigerant inlet pressure $\mathrm{p}_{\text {in }}=1.09 \div 1.17 \mathrm{MPa}$ (saturation temperature $\mathrm{t}_{\text {sat }}=42.6 \div 45.5^{\circ} \mathrm{C}$ ),

- Steady state and periodic hydrodynamic instabilities induced by a cut-valve for 40 different 
frequencies (the wave period between 0.1 and 4 seconds with 0.1 increment).

Figure 10 shows the correlation between the velocity of the pressure wave and the frequency of periodic instabilities for the R1234yf refrigerant and three different internal diameters of the minichannel. The shape of the curves agrees with the one for conventional channels shown in Figure 6. The frozen velocity in minichannels, however, is reached for much smaller frequencies. Both frozen and equilibrium velocities decrease with the internal diameter of the channel. There are many similarities between the R1234yf and R134a refrigerants in terms of pressure instabilities. The values of frozen velocities for both refrigerants are comparable, nevertheless, the equilibrium velocities for the R1234yf are substantially higher. For $\mathrm{ID}=3.3 \mathrm{~mm}$, the frozen velocity reaches $350 \mathrm{~m} / \mathrm{s}$, which is comparable with the speed of sound for normal conditions. Therefore, it is reasonable to regard this size as a transition between mini and conventional channels.
Figure 12 illustrates a correlation between the velocity of the condensation front and the frequency of periodic instabilities for the R1234yf refrigerant and the three different internal diameters of the minichannel. The curves also demonstrate similarities with one shown in Figure 6. Again the frozen velocity is reached for much smaller frequency and both asymptotes decrease with the internal diameter. However, their values are much closer to the ones for R134a shown in Figure 13.

Figure 14 represents the time-averaged values of wall temperature along the channel during periodic instabilities for the R1234yf refrigerants. The plateau that is clearly visible for the highest frequency represents the stable two-phase condensation zone. For smaller frequencies, the plateau is not present at all. This means that the high efficiency condensation zone was constantly moving along the channel. In the case of R134a (Figure 15), the position of the high efficiency condensation zone was more stable.

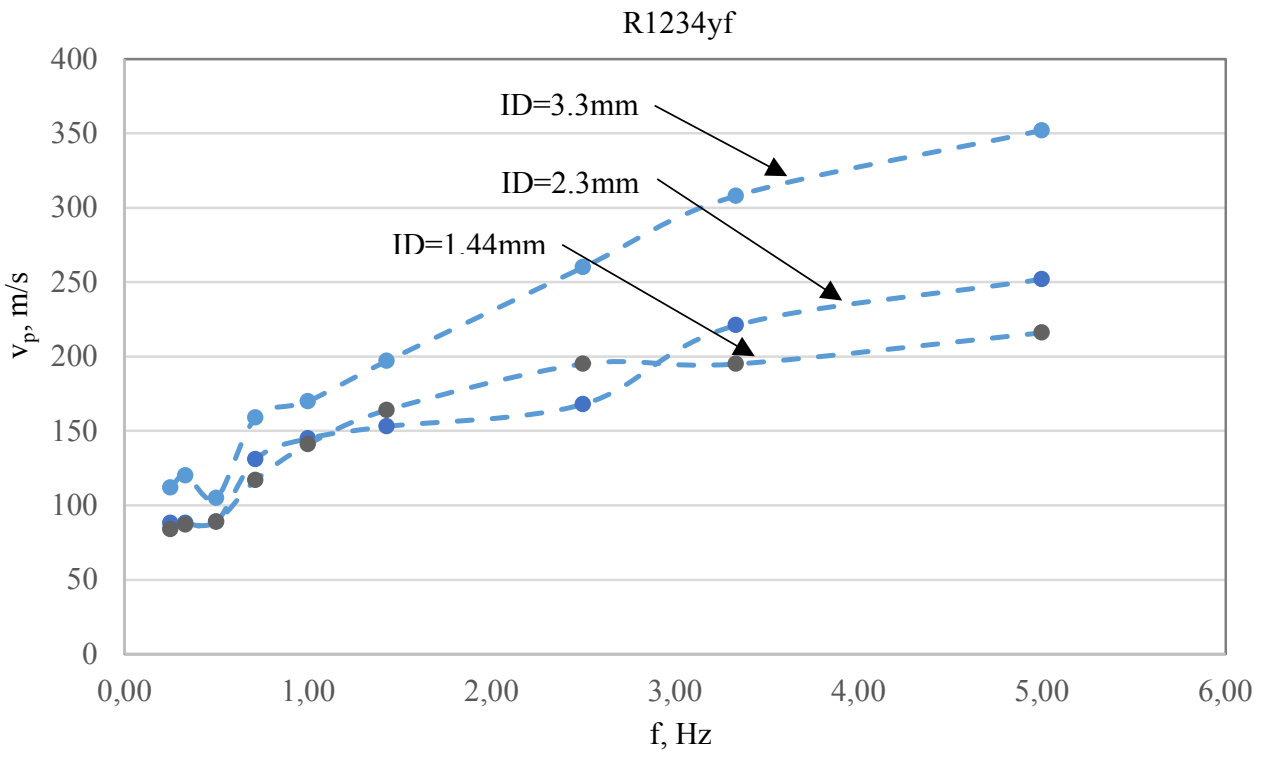

Fig. 10. Propagation velocity of pressure instability for R1234yf refrigerant and different IDs.

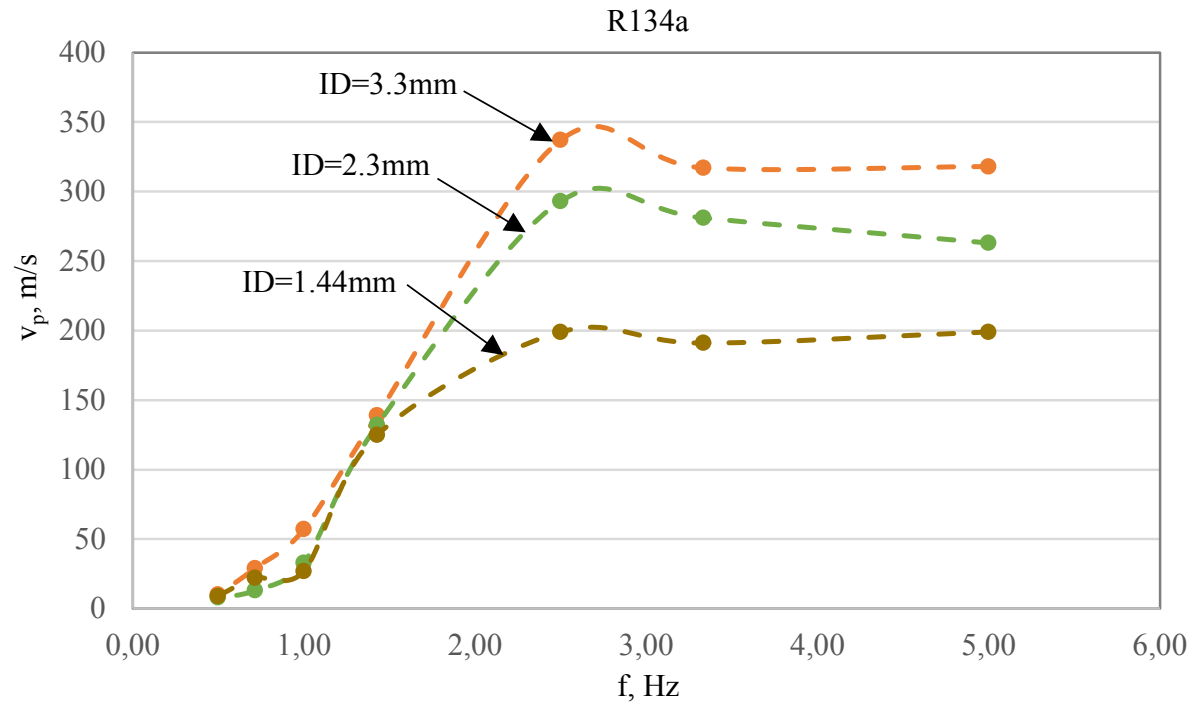

Fig. 11. Propagation velocity of pressure instability for R134a refrigerant and different IDs [8]. 


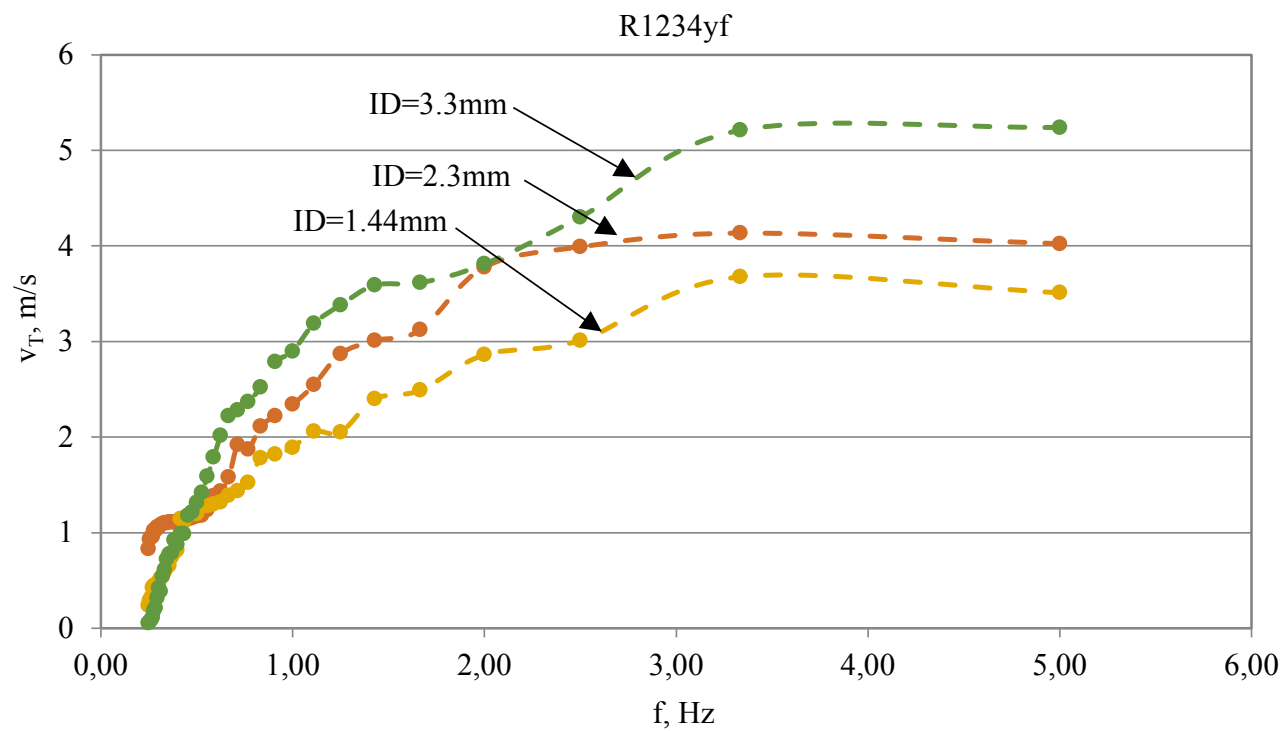

Fig. 12. Propagation velocity of temperature instability for R1234yf refrigerant and different IDs.

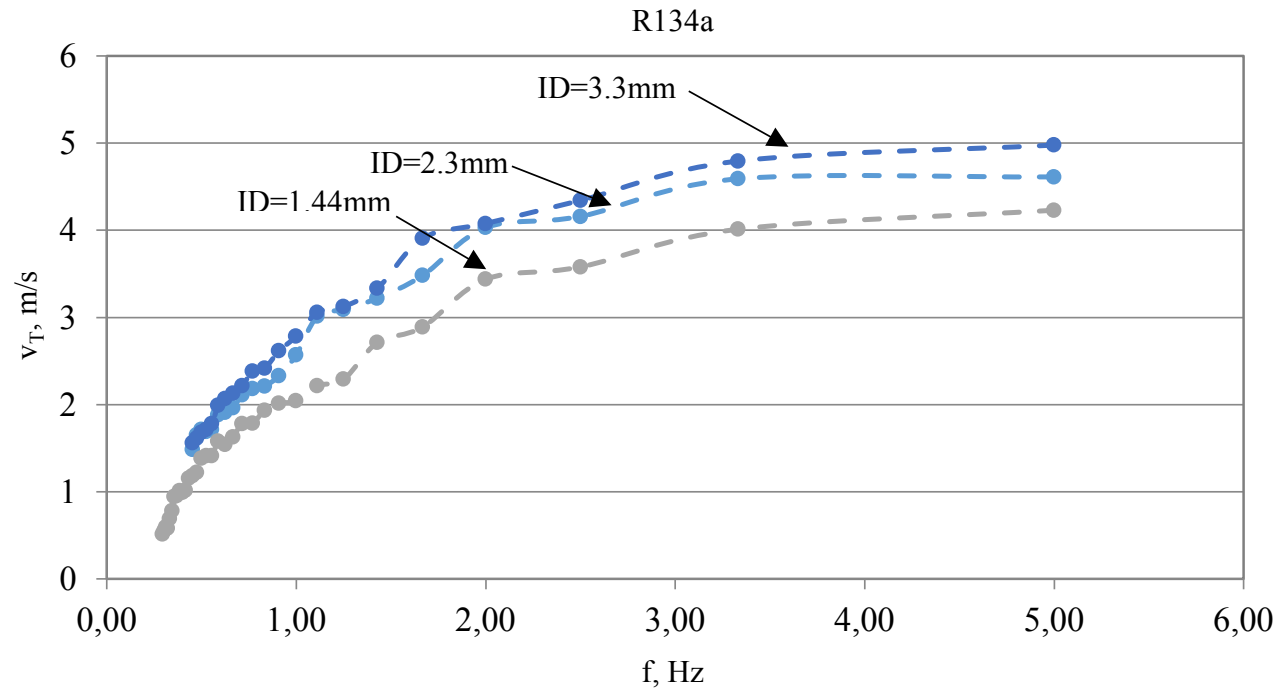

Fig. 13. Propagation velocity of temperature instability for R134a refrigerant and different IDs [8].

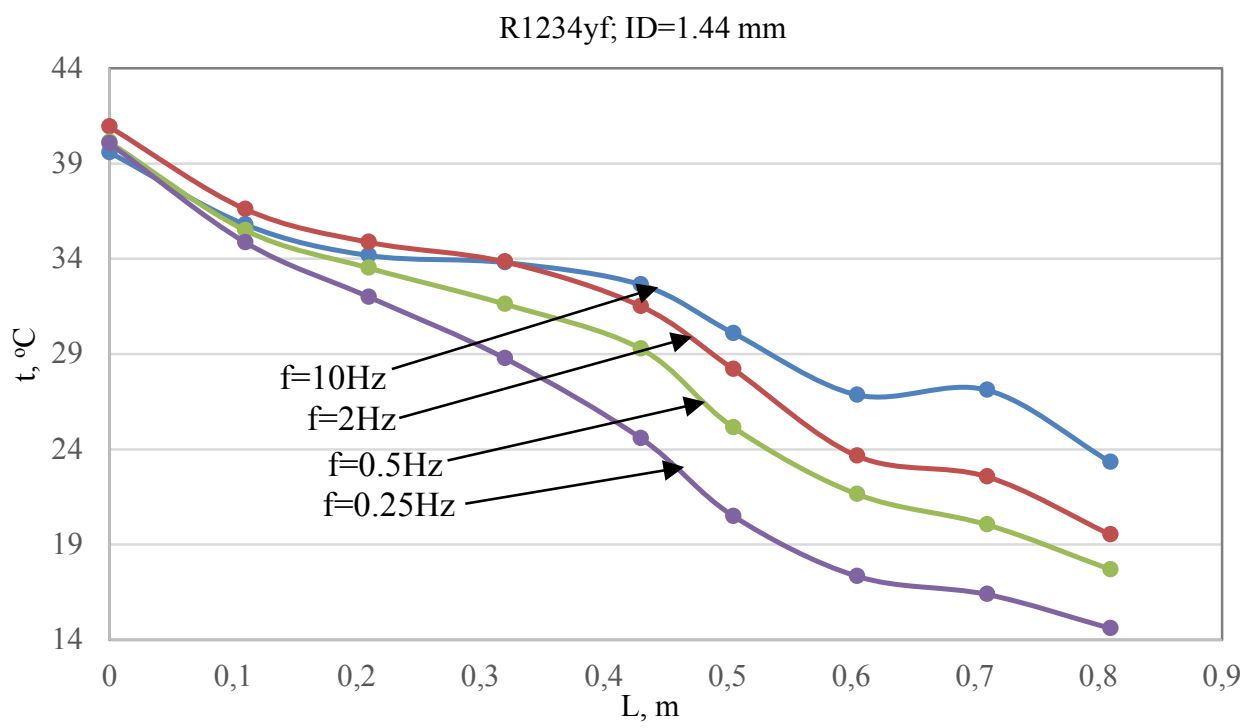

Fig. 14. Temperature distribution along the minichannel for R1234yf refrigerant $(\mathrm{ID}=1.44 \mathrm{~mm})$ and different frequencies of instabilities. 


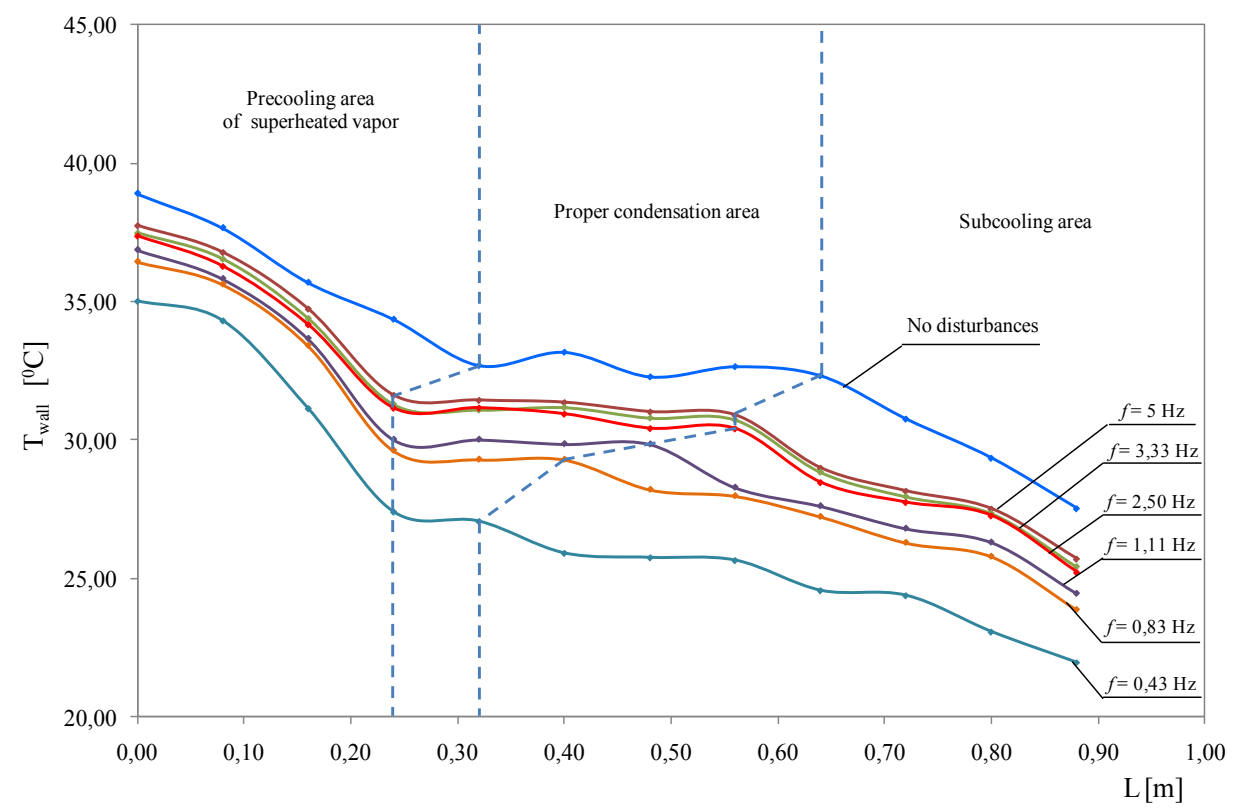

Fig. 14 Results of the experimental investigations concerning the influence of periodically generated instabilities on the length of the areas of superheat removal, proper condensation and subcooling of the R134a refrigerant depending on the frequency $f$ of the generated disturbances in minichannel with the internal diameter $d=1.44 \mathrm{~mm}$ [8].

\section{Conclusions}

An analysis of premilimary experimental research on hydrodynamic instabilities during condensation of the pro-ecological refrigerant R1234yf in tubular minichannels showed that:

1) For pressure instabilities, shape of the functions obtained for the R1234yf refrigerant (Figure 10) agree with the one for conventional channels (Figure 6). However, frozen velocity in minichannels is obtained for much smaller frequencies. The asymptotes tend to decrease for smaller IDs like in the previous studies made for the R134a refrigerant (Figure 11). Frozen velocities for the R1234yf refrigerant are comparable with those for R134a found in the literature, equilibrium velocities; however, they are significantly higher. ID $=3.3$ $\mathrm{mm}$ is transitional between conventional and minichannels, thus the pressure wave velocity is comparable with the speed of sound for normal conditions.

2) For temperature instabilities, the shape of the functions obtained for the R1234yf refrigerant (Figure 12) agrees with the one for conventional channels (Figure 6). However, the frozen velocity in minichannels is obtained for much smaller frequencies. The asymptotes tend to decrease for smaller IDs like in the previous studies made for the R134a refrigerant (Figure 13). Both frozen and equilibrium velocities for the R1234yf refrigerant are comparable with those for R134a found in the literature.

3) The high efficiency condensation zone shrank with a decrease of the frequency of the instabilities as expected (Figure 14). However, the plateau that was clearly visible during the investigation of the R134a refrigerant (Figure 15) did not occur due to a significant movement of the zone.

\section{References}

1. T. Bohdal, Bubble boiling in flow of refrigerating media. Journal of Mechanical and Energy Engineering, Vol. 1 No. 1 (41), June 2017, pp. 57 64.

2. T. Bohdal, M. Kruzel, M. Sikora, Analisys of heat transfer coefficient during refrigerant condensation in vertical pipe minichannel. Journal of Mechanical and Energy Engineering, Vol. 1 No. 1 (41), June 2017, pp. $65-70$

3. European Standard, PN-EN 14511:2009 Air conditioners, liquid chilling packages and heat pumps with electrically driven compressor for space heating and cooling

4. Polish Act, Act on the renewable Energy sources of $20^{\text {th }}$ February 2015, Dz.U z 2015 r., poz. 478 (in Polish)

5. Report, Bitzer - Refrigerants, edition no. 19, 2016 (in Polish)

6. S. Kandlikar, S. Garimella, D. Li, S. Colin, M.R. King, Heat transfer and fluid flow in minichannels and microchannels (2006)

7. S.S. Mehendale, A.M. Jacobi, R.K. Shah, Fluid flow and heat transfer at micro- and meso-scales with application to heat exchanger design, Applied Mechanics Rev. 53(2000), 7, pp. 175-193

8. W. Kuczyński, Hydrodynamic instabilities during condensation of refrigerants in minichannels, 2013 (in Polish)

9. K.A. Triplett, S.M. Ghiaasiaan, S.I. Abdel-Khalik, A. LeMouel, B.N. McCord, Gas-liquid two-phase flow in microchannels Part II: void fraction and 
pressure drop, Int. J. Multiphas Flow 25 (1999) pp. 395-410

10. Y.Y. Yan, T.F. Lin, Condensation heat transfer and pressure drop of refrigerant $R-134 a$ in a small pipe, Int. J. of Heat and Mass Transfer (1999) pp. 697 708

11. T. Nulboonrueng, J. Kaewon. S. Wongwises, Twophase condensation heat transfer coefficients of HFC-134a at high mass flux in smooth and microfin tubes, Int. Comm. Heat Mass Transfer 30 no. 4 (2003) pp. $577-590$

12. S.G. Kandlikar, Fundamental issue related to flow boiling in minichannels and micro-channels, Experimental Thermal and Fluid Mechanics and Thermodynamics, 2002, vol. 26, p. 129-146

13. H. Yuncu, O.T. Yildirim, S. Kakac, Two-phase flow instabilities in a horizontal single boiling channel Appl. Sci. Res. 48 (1991) pp. 83 - 104

14. J. Bouré, A. Bergles, L. Tong, Review of two-phase flow instabilities, Nucl. Eng. Des. 25 (1973) pp. 165-192

15. M. Ledinegg, Instability of flow during natural and forced circulation, Die Wärme 61 (8) (1938) pp. 891-898.

16. Y. Ding, S. Kakaç, Dynamic instabilities of boiling two-phase flow in a single horizontal channel, Experimental Thermal and Fluid Science (1995), pp. $327-342$

17. J. Maulbetsch, P. Griffith, A study of system-induced instabilities in forced convection flows with subcooled boiling, MIT engineering projects Lab Report 5382-35, 1965

18. K. Akagawa, T. Sakaguchi, Study on distribution of flow rates and flow stabilities in parallel long evaporates, Bull. JSME 14 (1971) pp. 837-848

19. Y. Buyevich, I. Natalukha, Self-oscillating regimes of nucleate, transition and film boiling, Int. J. Heat Mass Transfer 38 (2) (1996) pp. 2363-2373

20. A. Stenning, Instabilities in the flow of a boiling liquid, J. Basic Eng. (1964) pp. 213-217

21. T. Bohdal, M. Łomiak, Condensation of refrigeration medium under condition of unit disturbances, An International Journal of Turbulence, vol. 11, 2005, pp. 221 - 224

22. W. Kuczyński, T. Bohdal, H. Charun, Impact of periodically generated hydrodynamic disturbances on the condensation efficiency of R134a refrigerant in pipe mini-channel, Experimental Heat Transfer 26 (1), 2013, pp. 64 - 84.

23. W. Kuczyński, Modeling of the propagation of a pressure wave during the condensation process of
R134a refrigerant in a pipe minichannel under the periodic conditions of hydrodynamic disturbances, International Journal of Heat and Mass Transfer Volume 56, Issues 1-2, 1 January 2013, pp. 715723.

24. W. Kuczyński, T. Bohdal, H. Charun, Influence of hydrodynamic instability on the heat transfer coefficient during condensation of R134a and R404A refrigerants in pipe mini-channels, International Journal of Heat and Mass Transfer Volume 55, Issues 4, 1 January 2013, pp. 10831094.

25. Z. Bilicki, P. Downar-Zapolski, Wave phenomena related to two fluid and homogenous two-phase flow models, Prace INP PAN Gdańsk, no. 94, 1992, pp. 19 - 42 (in Polish)

26. R. Laskowski, M. Jaworski, Maximum entropy generation rate in a heat exchanger at constant inlet parameters. Journal of Mechanical and Energy Engineering, Vol. 1 No. 1 (41), June 2017, pp. $65-$ 70.

27. M. Sikora, Flow structures during refrigerants condensation. Journal of Mechanical and Energy Engineering, Vol. 1 No. 1 (41), June 2017, pp. 101 106.

28. T. Bohdal, M. Kruzel, M. Sikora, An investigation of heat transfer coefficient during refrigerants condensation in vertical pipe microchannel. Journal of Mechanical and Energy Engineering, Vol. 1(41) No. 2, November 2017, pp. 163 - 170.

29. W. Kuczynski, A. Denis, Modeling the instabilities of the condensation process of the R134a and R404A refrigerants in pipe minichannels in the conditions of periodic hydrodynamic disturbances. Journal of Mechanical and Energy Engineering, Vol. 1(41) No. 2, November 2017, pp. 179 - 188.

30. T. Bohdal, H. Charun, M. Sikora, Pressure drop during condensation of refrigerants in pipe minichannels, Archives of Thermodynamics (2012) 33(1), 87-106

31. T. Bohdal, K. Widomska, M. Sikora, The analysis of thermal and flow characteristics of the condensation of refrigerant zeotropic mixtures in minichannels, Archives of Thermodynamics (2016) 37(2), 41-69

32. T. Bohdal, H. Charun, M. Sikora, Heat transfer during condensation of refrigerants in tubular minichannels, Archives of Thermodynamics (2012) 33(2), 3-22

33. T. Bohdal, H. Charun, M. Sikora, Empirical study of heterogeneous refrigerant condensation in pipe minichannels, International Journal of Refrigeration (2015) 59, 210-223 